\title{
Association between extracellular volume control and survival in patients on short daily haemodialysis
}

\author{
Ana Beatriz Lesqueves Barra 1,2, Ana Paula Roque-da-Silva, ${ }^{1,2}$, Marcos S. Vasconcellos², Jocemir R. Lugon 1,3 \\ and Jorge Paulo Strogoff-de-Matos ${ }^{1,3^{*}}$ (i)
}

\begin{abstract}
Background: Fluid overload ( $F O$ ) assessed by bioimpedance spectroscopy $(\mathrm{BIS})$ is associated with higher mortality risk in maintenance haemodialysis (HD). The aim was to assess if a better management of FO through short daily haemodialysis (SDHD) could improve survival.
\end{abstract}

Methods: Retrospective analysis of patients who were on HD 3 sessions/week for at least 3 months and shifted to in-centre SDHD (5 or 6 sessions/week, 2 to 3 h/session) between July 2012 and June 2016 at 23 dialysis units in Brazil. The 12-month risk of death was analysed according to the predialysis hydration status measured before and 6 months after initiation of SDHD. Predialysis hydration status was considered adequate when FO $\leq 15 \%$ of extracellular volume.

Results: A total of 297 patients on SDHD were included in the analysis. Their median age was 57 (IQR 45-67) years, $62 \%$ were males, $44 \%$ diabetics, $57 \%$ on 6 dialysis sessions/week, with a median session duration of 130 (IQR 120150) minutes. BIS assessment at initiation of the SDHD regimen was performed in 220 patients and $F O>15 \%$ was found in $46.4 \%$. Twelve-month survival rates for those with FO $\leq 15$ and $>15 \%$ before initiating SDHD were 87.4 and $88.0 \%$, respectively $(P=0.92)$. BIS analysis when completing 6 months on SDHD were available for 229 patients, $26.6 \%$ with $\mathrm{FO}>15 \%$. The survival rates for the next 12 months (from the 6th to the 18th month of follow-up) for those with $\mathrm{FO} \leq 15$ and $>15 \%$ were 91.0 and $72.0 \%$, respectively $(P=0.0006)$. In a Cox regression model, after adjustment for demographic, clinical and laboratory variables, $\mathrm{FO} \leq 15 \%$ persisted associated with a lower mortality risk (hazard ratio $0.34,95 \% \mathrm{Cl} 0.13-0.87$ ).

Conclusions: Moving from conventional HD to SDHD was associated with better control of excessive extracellular volume. Patients who reached or maintained predialysis fluid overload $\leq 15 \%$ after initiating SDHD presented a lower risk of death.

Keywords: Dialysis, Short daily hemodialysis, Bioimpedance, Fluid overload, Survival analysis

\footnotetext{
* Correspondence: strogoff@uol.com.br

${ }^{1}$ Postgraduation Program in Medical Sciences, Fluminense Federal University (UFF), Niterói, Rio de Janeiro, Brazil

${ }^{3}$ Nephrology Division, Department of Medicine, Fluminense Federal

University (UFF), Av. Marques do Parana 303, 20 andar, Niteroi, Rio de Janeiro 24033-900, Brazil

Full list of author information is available at the end of the article
}

(c) The Author(s). 2020 Open Access This article is licensed under a Creative Commons Attribution 4.0 International License, which permits use, sharing, adaptation, distribution and reproduction in any medium or format, as long as you give appropriate credit to the original author(s) and the source, provide a link to the Creative Commons licence, and indicate if changes were made. The images or other third party material in this article are included in the article's Creative Commons licence, unless indicated otherwise in a credit line to the material. If material is not included in the article's Creative Commons licence and your intended use is not permitted by statutory regulation or exceeds the permitted use, you will need to obtain permission directly from the copyright holder. To view a copy of this licence, visit http://creativecommons.org/licenses/by/4.0/ The Creative Commons Public Domain Dedication waiver (http://creativecommons.org/publicdomain/zero/1.0/) applies to the data made available in this article, unless otherwise stated in a credit line to the data. 


\section{Background}

Mortality in maintenance haemodialysis (HD) patients is still unacceptably high, and cardiovascular disease continues to be the leading cause of death and hospitalizations in that population [1]. Although multiple factors inherent to renal disease are responsible for this risk, the intermittent nature of the conventional HD schedules may have an important contribution [2]. The establishment of HD prescription of a 3 to $4 \mathrm{~h}$ sessions, 3 times a week was not based on clinical outcomes. Such dialysis schedule was conceived in the 1970s to face economical and logistic challenges and devised to accommodate a greater number of patients with end-stage renal disease [3].

The standard HD many times does not fit the individual patient's needs, especially for appropriate fluid management. Short daily haemodialysis (SDHD) can improve extra-cellular volume management leading to a better blood pressure control and reduction of left ventricular mass as compared to the 3 -session per week HD regimen [4]. However, the estimation of HD patient dry weight using only clinical parameters is a challenge in clinical practice. Bioimpedance is a useful tool to improve the diagnosis of hydration status and the management of fluid in dialysis patients. Small clinical trials have shown that the use of bioimpedance to guide the volume management improved blood pressure control, decreased left ventricular mass and reduced mortality $[5,6]$.

Overhydration or fluid overload (FO), defined as the presence of more than $15 \%$ of the extracellular water, has been associated with higher mortality risk in patients on conventional HD [7-9]. Nevertheless, the association between fluid overload measured by bioimpedance and the risk of death in SDHD is not yet known. In the present study, we analyse if better management of overhydration through SDHD could have a positive impact on survival.

\section{Methods}

This is a retrospective analysis of adult patients $(\geq 18$ years old) from 23 dialysis facilities in Brazil on HD 3 sessions/week for at least three months who were shifted to in-centre SDHD between July 2012 and June 2016. The main indication for the modification of the dialysis schedule was inadequate fluid management on conventional HD diagnosed on clinical grounds. Due to limitations of more frequent HD reimbursement by the public health system, only patients with private health insurances were shifted to that HD regimen.

All demographic, clinical, laboratory and BIS data were extracted from the European Clinical Dialysis Database $\left(\mathrm{EuCliD}^{\circ}\right)$, a standardized electronic medical record used by all participating centres. This study was approved by the local ethics committee.
In all dialysis centres, the routine laboratory exams were performed monthly and blood samples were collected before the midweek dialysis session. BUN was also measured post-dialysis. Parameters of dialysis treatment and blood pressure values were defined as the mean of all measurements in the last 4 weeks. Dialysis adequacy was expressed as equilibrated $\mathrm{Kt} / \mathrm{V}$ for urea [10] and converted to weekly standard $\mathrm{Kt} / \mathrm{V}$ for patients on SDHD [11].

Body composition and hydration status were evaluated using a portable whole body bioimpedance spectroscopy (BIS) device $\left(\mathrm{BCM}^{\oplus}\right.$ - Body Composition Monitor, Fresenius Medical Care, Bad Homburg, Germany). BIS assessments were always performed immediately before dialysis sessions with the patient sitting in the dialysis chair. BIS assessment was recommended for all patients every 3 months, except in case of contraindication such as the use of metal hip prosthesis. Adequate hydration was defined as a relative FO/extracellular volume below $15 \%$.

The last BIS evaluation before increasing the frequency of treatment was chosen to define the hydration status before SDHD. The last BIS evaluation before completing the 6th month on SDHD was taken to define the hydration status after SDHD.

Three different 12-month survival analyses were accomplished. First, we looked at survival from month 0 to 12 according to the hydration status (FO $\leq 15 \%$ versus > $15 \%)$ before the change to SDHD. Second, we analysed survival from month 6 to 18 but considering the hydration status (FO $\leq 15 \%$ versus $>15 \%$ ) after at least three months on SDHD. Finally, four survival curves were compared according to the individual variation of the hydration status ( $\Delta \mathrm{FO}$ ) before and after moving to SDHD: patients who remained with adequate extracellular volume control (adequate - adequate); patients who were initially overloaded but became normohydrated (high - adequate); patients who were fluid overloaded and so remained after switching to SDHD (high - high); and patients who eventually had a deterioration of fluid control on SDHD (adequate - high), Fig. 1.

\section{Statistical analysis}

Kolmogorov-Smirnov was used to test for the distribution of variables. Variables with normal distribution were expressed as mean \pm standard deviation; those with nonGaussian distribution, as median and interquartile intervals. T-test or Mann-Whitney was used as appropriate when two groups were compared. The Kaplan-Meier method was used for the analysis of survival, and comparison between curves was made by the Log-Rank test. Overhydration as a predictor of death risk was analysed in a multivariate Cox regression model. In all analysis, $P<0.05$ were considered significant. The software SPSS, 


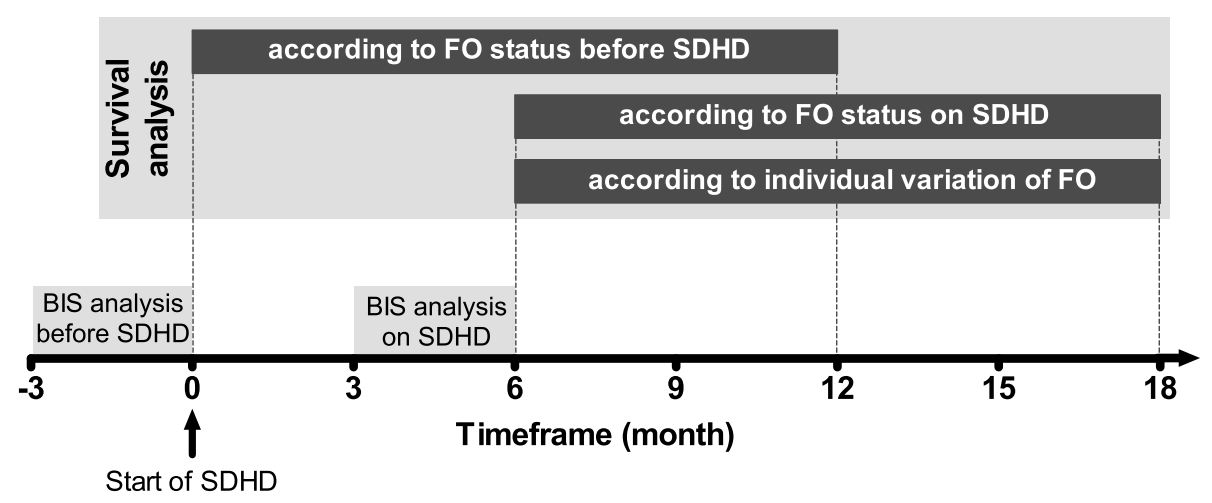

Fig. 1 Design of survival analysis according to the FO status before and after starting SDHD. Schematization of the three different survival analysis: according to the fluid overload (FO) status assessed by bioimpedance spectroscopy (BIS) before starting short daily haemodialysis (SDHD); FO status after switching to SDHD; or the individual variation of FO status comparing BIS analysis before and after starting SDHD

Table 1 Profile of patients whereas on conventional haemodialysis immediately before shifting to short daily haemodialysis

\begin{tabular}{|c|c|c|c|c|}
\hline \multirow[t]{2}{*}{ Variables } & All patients & $\mathrm{FO} \leq 15 \%$ & $\mathrm{FO}>15 \%$ & $P$ value \\
\hline & $(n=297)$ & $(n=118)$ & $(n=102)$ & \\
\hline$\overline{\mathrm{FO}}(\% \mathrm{ECV})^{\S}$ & $13.8(5.1-22.1)$ & $6.2(1.9-10.4)$ & $23.9(17.8-30.0)$ & - \\
\hline Age (years) & $57(45-67)$ & $53(39-63)$ & $61(48-70)$ & $<0.0001$ \\
\hline Male gender, n (\%) & $189(63.6)$ & $70(59.3)$ & $70(68.6)$ & 0.16 \\
\hline Diabetes, n (\%) & $128(43.1)$ & $43(36.4)$ & $52(51.0)$ & 0.04 \\
\hline Charlson comorbidity index & $4(2-4)$ & $3(2-4)$ & $4(2-4)$ & 0.018 \\
\hline Vintage (months) & $29(13-61)$ & $33(13-61)$ & $29(16-70)$ & 0.38 \\
\hline $\mathrm{HBsAg}+, \mathrm{n}(\%)$ & $3(1.0)$ & $1(0.8)$ & $1(1.0)$ & 1.00 \\
\hline Anti-HCV+, n (\%) & $9(3.0)$ & $4(3.4)$ & $3(3.0)$ & 1.00 \\
\hline Anti-HIV+, n (\%) & $6(2.0)$ & $4(3.4)$ & $1(1.0)$ & 0.39 \\
\hline \multicolumn{5}{|l|}{ Vascular access, n (\%) } \\
\hline Native AFV & $210(70.7)$ & $97(82.2)$ & $69(67.6)$ & 0.018 \\
\hline Graft & $13(4.4)$ & $6(5.1)$ & $2(2.0)$ & 0.29 \\
\hline Catheter & $74(24.9)$ & $15(12.7)$ & $31(30.4)$ & 0.002 \\
\hline Treatment time (min/session) & $240 \pm 10$ & $239 \pm 10$ & $240 \pm 10$ & 0.36 \\
\hline Equilibrated KTN & $1.28 \pm 0.35$ & $1.29 \pm 0.37$ & $1.27 \pm 0.33$ & 0.81 \\
\hline Body mass index $\left(\mathrm{Kg} / \mathrm{m}^{2}\right)$ & $25.5 \pm 5.6$ & $26.8 \pm 6.2$ & $24.0 \pm 5.0$ & $<0.0001$ \\
\hline Body weight (Kg) & $71.0 \pm 17.3$ & $74.8 \pm 18.8$ & $67.3 \pm 15.1$ & 0.0003 \\
\hline UF rate (mL/hour/Kg) & $9.9 \pm 3.5$ & $9.9 \pm 3.4$ & $10.1 \pm 3.6$ & 0.71 \\
\hline Predialysis SBP (mmHg) & $139 \pm 21$ & $138 \pm 19$ & $141 \pm 22$ & 0.19 \\
\hline Predialysis DBP (mmHg) & $76 \pm 13$ & $77 \pm 12$ & $74 \pm 14$ & 0.018 \\
\hline Postdialysis SBP (mmHg) & $134 \pm 20$ & $132 \pm 18$ & $138 \pm 22$ & 0.008 \\
\hline Postdialysis DBP (mmHg) & $75 \pm 12$ & $76 \pm 12$ & $74 \pm 13$ & 0.12 \\
\hline Haemoglobin (g/dL) & $10.5 \pm 1.9$ & $10.9 \pm 1.8$ & $10.1 \pm 1.9$ & 0.002 \\
\hline Serum albumin $(g / L)$ & $37.7 \pm 4.8$ & $39.1 \pm 4.0$ & $37.0 \pm 5.2$ & 0.0002 \\
\hline Phosphorus (mg/dL) & $5.1 \pm 1.6$ & $5.3 \pm 1.5$ & $5.1 \pm 1.7$ & 0.24 \\
\hline
\end{tabular}

Data are expressed as frequency (\%), mean \pm standard deviation or median (interquartile interval)

FO fluid overload; ECV extracellular volume; AVF arteriovenous fistula; UF ultrafiltration; SBP systolic blood pressure; $D B P$ diastolic blood pressure; ${ }^{\S}$ Data available in only 220 patients 
version 21.0 (Chicago, Illinois, USA) was used for the statistical analysis.

\section{Results}

A total of 297 patients on SDHD were included in the analysis. The characteristics of the patients are presented in Table 1. BIS assessment before initiating SDHD was performed in 220 patients and $102(46.4 \%)$ of them had initial FO $>15 \%$. Patients with FO $>15 \%$ before initiating SDHD was older, had a higher prevalence of diabetes and central intravenous catheter as the vascular access, and lower levels of serum albumin than the ones with FO $\leq 15 \%$ (Table 1). The survival rates for the first 12month on SDHD were $87.4 \%$ for those with FO $\leq 15$ and $88.0 \%$ for those with FO $>15 \%(P=0.92)$, Fig. 2 .

After 6 months, 256 patients remained on SDHD. By that time, the mean blood pressure levels were reduced, and the mean serum albumin, bicarbonate and haemoglobin were higher with no significant change in serum phosphate (Table 2).

Data from BIS analysis before completing 6 months on SDHD were available for 229 out of 256 patients. Median FO was reduced from 13.8\% (interquartile range [IQR] 5.1-22.1\%) to 7.6\% (IQR $-0.4-15.3 \%$ ), $P<0.0001$ (Table 2) and the rate of patients with $\mathrm{FO}>15 \%$ dropped from 46.4 to $26.6 \%(P=0.0005)$.

\section{Hydration status on SDHD and survival}

The survival rates from the 6th to the 18th month on SDHD, for those with FO $\leq 15 \%$ or $>15 \%$ before the start of this timeframe were 91 and $72 \%$, respectively ( $P=$ 0.0006), Fig. 3. Patients with FO $>15 \%$ on SDHD tended to be older, with a predominance of the male gender.
Table 2 Changes in body composition by BIS assessment and selected laboratory parameters from the first to the sixth month on short daily haemodialysis

\begin{tabular}{|c|c|c|c|}
\hline Variables & $\begin{array}{l}\text { Month } 0 \\
(n=297)\end{array}$ & $\begin{array}{l}\text { Month } 6 \\
(n=256)\end{array}$ & $P$ value \\
\hline$\overline{\mathrm{FO}}(\% \mathrm{ECV})^{\S}$ & $13.8(5.1-22.1)$ & $7.6(-0.4-15.3)$ & $<0.0001$ \\
\hline Fat mass $(\%)^{\S}$ & $36(27-43)$ & $38(29-44)$ & 0.13 \\
\hline Lean mass $(\%)^{\S}$ & $47(39-59)$ & $46(37-56)$ & 0.38 \\
\hline Dry weight (Kg) & $71.0 \pm 17.3$ & $71.2 \pm 16.6$ & 0.91 \\
\hline Predialysis SBP (mmHg) & $139 \pm 21$ & $133 \pm 21$ & 0.0011 \\
\hline Predialysis DBP (mmHg) & $76 \pm 13$ & $72 \pm 12$ & 0.0004 \\
\hline Postdialysis SBP (mmHg) & $134 \pm 20$ & $129 \pm 20$ & 0.0018 \\
\hline Postdialysis DBP (mmHg) & $75 \pm 12$ & $72 \pm 12$ & 0.0016 \\
\hline Haemoglobin (g/dL) & $10.5 \pm 1.9$ & $10.9 \pm 2.0$ & 0.050 \\
\hline Serum albumin (g/L) & $37.7 \pm 4.8$ & $39.6 \pm 4.1$ & $<0.0001$ \\
\hline Predialysis BUN (mg/dL) & $57.3 \pm 19.4$ & $53.6 \pm 17.9$ & 0.035 \\
\hline Phosphorus (mg/dL) & $5.1 \pm 1.6$ & $5.1 \pm 1.5$ & 0.24 \\
\hline Calcium (mg/dL) & $9.1 \pm 0.8$ & $9.1 \pm 0.8$ & 0.76 \\
\hline Potassium (mEq/L) & $5.2 \pm 0.9$ & $5.1 \pm 0.8$ & 0.19 \\
\hline Sodium (mEq/L) & $138 \pm 4$ & $137 \pm 5$ & 0.29 \\
\hline HCO3- (mEq/L) & $20.9 \pm 2.9$ & $22.4 \pm 3.0$ & 0.031 \\
\hline
\end{tabular}

Data are expressed as mean \pm standard deviation or median (interquartile interval)

FO fluid overload; $E C V$ extracellular volume; $B U N$ blood urea nitrogen; SBP systolic blood pressure; DBP diastolic blood pressure; § Data available in only 220 patients at month 0 and 229 patients at month 6

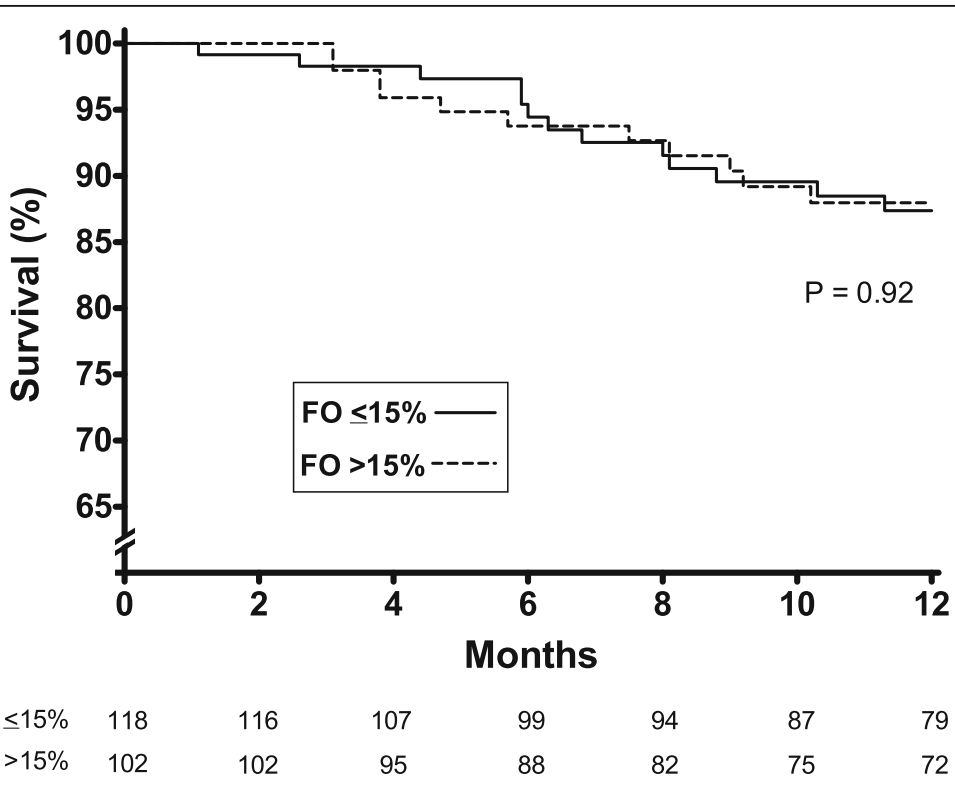

Fig. 2 Survival curves on SDHD according to the hydration status before initiating SDHD 


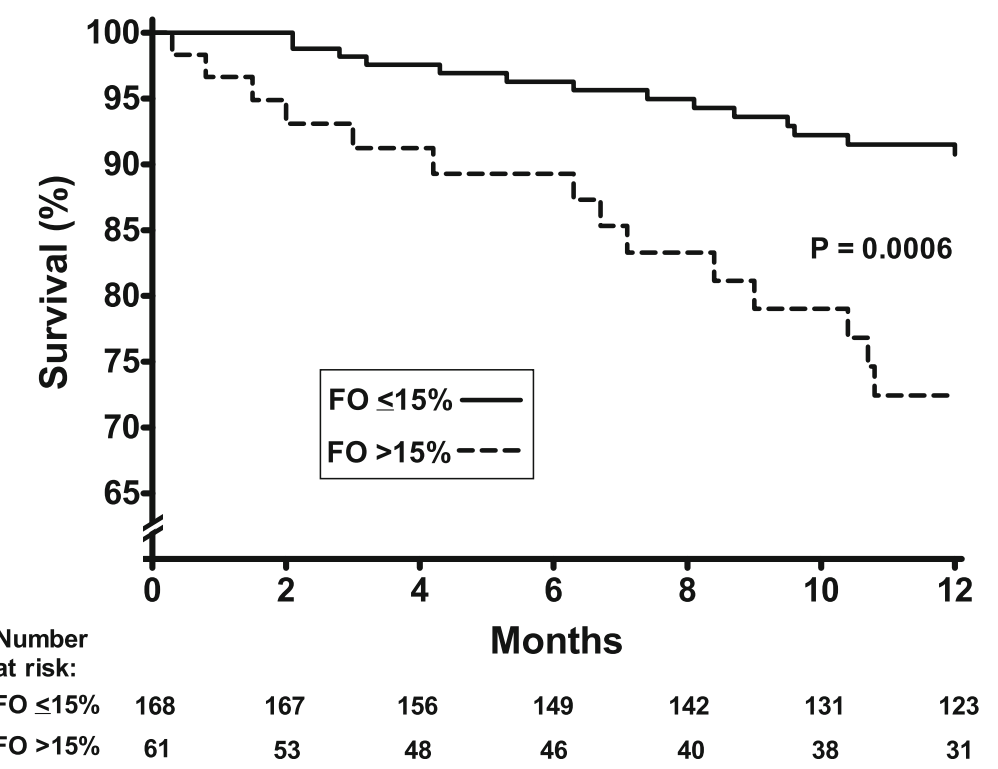

Fig. 3 Survival curves on SDHD according to the hydration status after switching to SDHD. Survival curves from 6th to 18th month on short daily haemodialysis (SDHD) according to the hydration status after switching to SDHD

They also presented a lower body mass index, serum albumin and haemoglobin (Table 3).

In a Cox regression model, the crude mortality risk was reduced for patients with $\mathrm{FO} \leq 15 \%$ (hazard ratio 0.30, 95\%CI 0.14-0.62). After adjustment for demographic, clinical and laboratory variables, FO $\leq 15 \%$ on SDHD persisted associated with lower mortality risk (hazard ratio $0.34,95 \% \mathrm{CI} 0.13-0.87$ ). In the same analysis, older age, low serum albumin and predialysis systolic blood pressure below $130 \mathrm{mmHg}$ were associated with higher mortality risk (Table 4).

\section{Change in hydration status after shifting to SDHD}

One hundred and seventy-eight had both BIS assessments (before and after initiating SDHD) allowing evaluation of their change in the hydration status. Taking the hydration status of the two studied periods, patients were distributed in following categories: $81(46 \%)$ patients were in the adequate - adequate; 13 (7\%) in the adequate - high; 38 (21\%) in the high - high; and 46 (26\%) in the high - adequate.

The survival rate from the 6th to 18th month on SDHD was $89 \%$ for patients with adequate hydration status on SDHD regardless of their hydration status on conventional HD, Fig. 4. On the other hand, for patients who either persisted with a high hydration status or switched from an initially adequate hydration status to a worse control after initiation of SDHD, the survival rate was poorer ( 74 and $58 \%$, respectively).

\section{Discussion}

Our findings suggest that SDHD can improve volume control in many patients who were not previously well controlled in the conventional HD regimen of 3 sessions per week. Maintaining or reaching a hydration status up to $15 \%$ above the normal volume, as estimated for the extracellular fluid measured by bioimpedance, was associated with a significant reduction in the risk of death in the following 12 months. Such finding is suggestive that the same cut-off point, used in previous studies with patients on conventional HD, could also be applied for patients on SDHD [7, 8]. Recently, Zoccali et al. [9] analysed the association between hydration status and death risk in almost 40 thousand HD patients, with more 200,000 BIS evaluations. Overhydration was strongly associated with the risk of death. In that study, inadequate hydration status was defined as FO > 15\% for men and $>13 \%$ for women.

Wizemann et al. [7] found a more than 2-fold increase in the risk of death in 269 patients on conventional HD who were fluid overloaded at the beginning of a 3. 5year follow-up period. In that study, only $22 \%$ of the patients were overloaded (FO $>15 \%$ ), a number that contrasts with our sample in which FO $>15 \%$ was found in $46 \%$ of patients before moving to SDHD. Shifting to SDHD regimen could be seen as a strategy to improve patients' hydration status [4]. Indeed, only $26.6 \%$ of our patients were still fluid overloaded after 6 months on SDHD. As the majority of them had been on conventional HD for more than a year before moving to SDHD, with a median of 29 months on renal replacement therapy, those patients probably would not have had any improvement in their hydration status if they had been maintained in the same regimen of HD 3 times per week. 
Table 3 Characteristics of patients at the 6th month on short daily haemodialysis according to the hydration status

\begin{tabular}{|c|c|c|c|}
\hline Variables & $\begin{array}{l}\text { FO } \leq 15 \% \\
(n=168)\end{array}$ & $\begin{array}{l}\mathrm{FO}>15 \% \\
(n=61)\end{array}$ & $P$ value \\
\hline FO (\%ECV) & $3.5(-2.4-9.0)$ & $22.3(17.9-27.1)$ & - \\
\hline Age (years) & $55(41-66)$ & $61(47-73)$ & 0.086 \\
\hline Male gender (\%) & 57.7 & 70.5 & 0.092 \\
\hline Diabetes (\%) & 37.5 & 49.2 & 0.13 \\
\hline Charlson comorbidity index & $3(2-4)$ & $4(2-4)$ & 0.066 \\
\hline Vintage (months) & $28(11-60)$ & $39(17-70)$ & 0.13 \\
\hline \multicolumn{4}{|l|}{ Vascular access (\%) } \\
\hline Native AFV & 74.4 & 62.3 & 0.098 \\
\hline Graft & 3.0 & 8.2 & 0.14 \\
\hline Catheter & 22.6 & 29.5 & 0.30 \\
\hline \multicolumn{4}{|l|}{ HD frequency (\%) } \\
\hline 5 sessions/week & 41.1 & 55.7 & 0.052 \\
\hline 6 sessions/week & 58.9 & 44.3 & \\
\hline Treatment time (min/week) & $814 \pm 189$ & $779 \pm 139$ & 0.19 \\
\hline Standard KTN & $3.45 \pm 1.01$ & $3.15 \pm 0.85$ & 0.067 \\
\hline Body mass index $\left(\mathrm{Kg} / \mathrm{m}^{2}\right)$ & $26.1 \pm 5.8$ & $23.6 \pm 5.4$ & 0.003 \\
\hline Dry weight (Kg) & $72.0 \pm 16.4$ & $68.9 \pm 17.1$ & 0.21 \\
\hline UF rate (mL/hour/Kg) & $10.8 \pm 3.2$ & $10.5 \pm 3.8$ & 0.58 \\
\hline Pre-HD SBP (mmHg) & $133 \pm 21$ & $133 \pm 21$ & 0.96 \\
\hline Pre-HD DBP (mmHg) & $73 \pm 11$ & $69 \pm 14$ & 0.053 \\
\hline Post-HD SBP (mmHg) & $128 \pm 19$ & $133 \pm 22$ & 0.086 \\
\hline Post-HD DBP (mmHg) & $72 \pm 11$ & $70 \pm 14$ & 0.18 \\
\hline Haemoglobin (g/dL) & $11.2 \pm 2.0$ & $10.1 \pm 1.8$ & 0.0002 \\
\hline Serum albumin (g/L) & $40.0 \pm 3.7$ & $38.3 \pm 4.6$ & 0.004 \\
\hline Phosphorus (mg/dL) & $5.3 \pm 1.5$ & $4.8 \pm 1.3$ & 0.062 \\
\hline
\end{tabular}

Data are expressed as mean \pm standard deviation or median (interquartile interval)

FO, fluid overload; ECV, extracellular volume; AVF, arteriovenous fistula; UF, ultrafiltration; SBP, systolic blood pressure; DBP, diastolic blood pressure

The intermittent nature of the in-centre conventional HD schedules could have a relevant role in the high mortality rate in the dialysis population. Two factors related to volume overload may contribute to a worse prognosis: the higher left ventricular preload related to the long periods of fluid accumulation, favouring left ventricular hypertrophy, and the excessive ultrafiltration rate $[12,13]$. Left ventricular hypertrophy is associated with high all-cause and cardiovascular mortality in dialysis patients and is usually used as a surrogate marker of a dismal course in dialysis. In the Frequent Hemodialysis Network Daily Trial, SDHD led to a significant reduction of left ventricular mass [4].

In the present study, switching to a more frequent treatment also impacted positively on blood pressure control, serum bicarbonate, albumin and haemoglobin.
Table 4 Cox regression models for prediction of one-year death risk in 229 patients with bioimpedance assessment at the 6th month on short daily haemodialysis by levels of adjustment

\begin{tabular}{llll}
\hline Variables & Model 1 & Model 2 & Model 3 \\
& Hazard ratio (95\% confidence interval) \\
\hline FO $\leq 15 \%$ & $0.30(0.14-0.62)$ & $0.26(0.11-0.61)$ & $0.34(0.13-0.87)$ \\
Age (year) & - & $1.05(1.02-1.09)$ & $1.05(1.02-1.08)$ \\
Male gender & - & $0.82(0.36-1.88)$ & $1.0(0.39-2.55)$ \\
Diabetes & - & $1.30(0.55-3.05)$ & $1.47(0.59-3.68)$ \\
Vintage (month) & - & $1.00(0.99-1.01)$ & $1.00(0.99-1.01)$ \\
Catheter & - & $1.49(0.59-3.75)$ & $1.49(0.59-3.75)$ \\
BMl (Kg/m $\left.{ }^{2}\right)$ & - & $1.06(0.98-1.15)$ & $1.09(0.99-1.19)$ \\
SBP $<130 \mathrm{mmHg}$ & - & $2.06(0.93-4.59)$ & $2.46(1.06-5.69)$ \\
UF rate (mL/h/Kg) & - & $1.06(0.92-1.23)$ & $1.07(0.92-1.25)$ \\
Standard KTN & - & - & $1.01(0.47-2.16)$ \\
Serum albumin (g/L) & - & - & $0.88(0.79-0.99)$ \\
Phosphorus (mg/dL) & - & - & $0.79(0.56-1.10)$ \\
Haemoglobin (g/dL) & - & - & $0.95(0.76-1.19)$ \\
\hline
\end{tabular}

Model 1: unadjusted hazard ratio, Model 2: adjustment for clinical and demographics; Model 3: model 2 plus laboratory variables. FO, fluid overload; $B M I$, body mass index; SBP, systolic blood pressure; UF, ultrafiltration

On the other hand, no improvement in the serum phosphorus control was seen and only a modest reduction in predialysis BUN was found 6 months after starting SDHD. We hypothesize that this could be a consequence of improved appetite and, consequently, increased protein intake.

For decades, the concept of dry weight in HD has been accepted as the weight that keeps patient normotensive without the need of hypotensive drugs. In fact, appropriate management of imbalanced fluid status can adequately control blood pressure in many cases [14]. However, determining dry weight based on trial and error is particularly challenging to hypotensive patients who are simultaneously hypervolemic. In this study, we found that predialysis systolic blood pressure below 130 $\mathrm{mmHg}$ was independently associated with a more than 2 -fold increase in the risk of death. As seen by Zoccali et al. [9], the risk of death was higher in the subgroup of patients who presented overhydration concomitant to predialysis systolic blood pressure below $130 \mathrm{mmHg}$, compared to the group without overhydration and predialysis systolic blood pressure between 130 and 160 mmHg.

Just recently, complementary methods for assessing volume status, such as BIS and lung ultrasound, have turned the search for dry weight less subjective. We found that even after moving to a more frequent treatment and having BIS as an accessory tool, many patients persisted with excessive overhydration and had reduced survival rate. It is hard to determine whether this reflects 


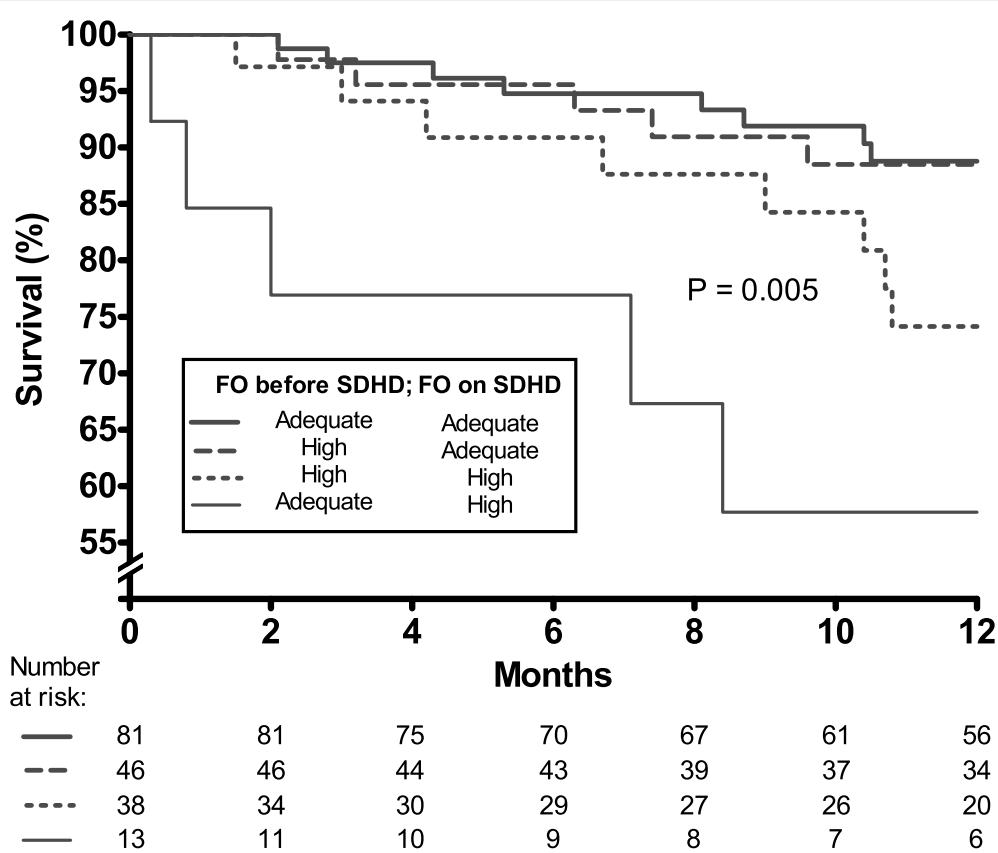

Fig. 4 Survival curves on SDHD according to the hydration status before and after switching to SDHD. Survival curves from 6th to 18th month on short daily haemodialysis (SDHD) according to the combination of hydration status in two different occasions (before and after switching to SDHD)

unfavourable clinical conditions or simply if the overhydration condition was overlooked and the patients were not properly managed after moving to SDHD. In the latter hypothesis, the opportunity for appropriate intervention in a modifiable variable with the potential to improve the prognosis could have been missed.

The deterioration of hydration status after moving to SDHD was only seen in 13 patients. They had adequate hydration control on conventional HD but unexpectedly deteriorated their hydration status after moving to SDHD. That subgroup of patients had a very poor outcome, with only $58 \%$ survival rate at 12 months. It is possible that the worsening of those patients could be a consequence of a decline in their clinical condition, from causes not related to the treatment, leading to a weight loss not promptly recognized and without the due adjustment in their dry weight.

Another method to diagnose overhydration in haemodialysis patients is the B-lines score through the lung ultrasound. Zoccali et al. [15] found moderate-to-severe lung congestion in $45 \%$ and very severe congestion in $14 \%$ of patients on $\mathrm{HD}$, and the vast majority of them was asymptomatic or presented slight symptoms of heart failure. Those patients with very severe congestion had more than 4-fold risk of death than the patients having mild or no congestion. There is an ongoing clinical trial designed to evaluate the effects of dry weight adjustment guided by lung ultrasound on clinical outcomes [16]. Interim results showed an effective and safe reduction of ambulatory blood pressure levels [17]. A limitation of lung ultrasound in the daily clinical practice is the dependence of a trained doctor for its execution [18]. Thus, compared to lung ultrasound, BIS is an easy-to-perform tool which can be used as many times as indicated.

In-centre SDHD is a well-established modality of therapy in the literature, which is associated with improved health related quality of life as compared to conventional haemodialysis [19], but it does require that patients have to attend the dialysis centre more frequently. In this regard, there may be room for home haemodialysis that could offer the benefits reported in the present study with more autonomy and perhaps a better quality of life [20].

Our study presents several limitations, including the retrospective nature of the analysis and missing BIS assessment data for a significant number of patients, both at baseline and the 6th month on SDHD. Other weakness of the study is the lack of uniformity regarding the interdialytic interval preceding the BIS assessment. Patients underwent BIS assessment always immediately before the dialysis session, but there was no control of the day of the week in which it was performed or the interdialytic interval that preceded the data collection. Finally, adjustments could only be performed by confounders that were available in the database, a limitation inherent to retrospective studies. The study also has its strengths, such as the inclusion of a large number of patients and the standardization of procedures, evaluations and register of data in all dialysis facilities. 


\section{Conclusion}

Moving from conventional haemodialysis to SDHD was associated with better control of excessive extracellular volume and blood pressure, and improvement in several laboratory parameters. Patients who reached adequate hydration control after initiating SDHD, i.e., up to $15 \%$ above the normal volume estimated for the extracellular fluid, presented a lower risk of death. Thus, pursuing an adequate extracellular volume control by intensifying haemodialysis frequency can be a strategy to manage a variable that impacts on survival.

\section{Abbreviations}

BIS: Bioimpedance spectroscopy; FO: Fluid overload; HD: Haemodialysis; SDHD: Short daily haemodialysis

\section{Authors' contributions}

A.B.B. was involved with data collection and organization, analysis of the data and manuscript writing. A.P.R.S. was involved with data collection, organization and analysis of the data. M.S.V. was involved with data collection, organization and development of study design. J.R.L. helped with study design, data interpretation, and manuscript writing. J.P.S.M. conceived the study and was involved with every step of the project. All authors have read and approved the manuscript.

\section{Funding}

This study was supported by Coordenação de Aperfeiçoamento de Pessoal de Nível Superior (CAPES), Ministry of Education, Brazil.

\section{Availability of data and materials}

The datasets used and analysed during the current study are available from the corresponding author on reasonable request

\section{Ethics approval and consent to participate}

Project approved by the research ethics committee of the Department of Medicine, Universidade Federal Fluminense, Niterói, RJ, Brazil. Approved Project Number: CAAE 76623317.10000.5243. No written informed consent was requested due to the retrospective nature of the study.

\section{Consent for publication}

Not applicable.

\section{Competing interests}

A.B.B., A.P. R.S. and M.S.V. are Fresenius Medical Care Brasil employees J.P.S.M received consultant fees from Fresenius Medical Care Brasil.

J.R.L declares no conflict of interest.

\section{Author details}

${ }^{1}$ Postgraduation Program in Medical Sciences, Fluminense Federal University (UFF), Niterói, Rio de Janeiro, Brazil. Fresenius Medical Care Brasil, Rio de Janeiro, Brazil. ${ }^{3}$ Nephrology Division, Department of Medicine, Fluminense Federal University (UFF), Av. Marques do Parana 303, 20 andar, Niteroi, Rio de Janeiro 24033-900, Brazil.

Received: 8 January 2020 Accepted: 20 April 2020

Published online: 29 April 2020

\section{References}

1. United States Renal Data System. 2018 USRDS annual data report: Epidemiology of kidney disease in the United States. National Institutes of Health, National Institute of Diabetes and Digestive and Kidney Diseases, Bethesda, MD, 2018

2. Foley RN, Gilbertson DT, Murray J, Collins AJ. Long Interdialytic interval and mortality among patients receiving hemodialysis. N Engl J Med. 2011;365: 1099-107.

3. Scribner BH, Cole JJ, Ahmad S, Blagg CR. Why thrice weekly dialysis? Hemodial Int. 2004;8:188-92.
4. FHN Trial Group, Chertow GM, Levin NW, Beck GJ, et al. In-center hemodialysis six times per week versus three times per week. N Engl J Med. 2010;363:2287-300.

5. Hur E, Usta M, Toz H, et al E. Effect of fluid management guided by bioimpedance spectroscopy on cardiovascular parameters in hemodialysis patients: a randomized controlled trial. Am J Kidney Dis 2013; 61:957-965.

6. Onofriescu M, Hogas S, Voroneanu L, et al. Bioimpedance-guided fluid management in maintenance hemodialysis: a pilot randomized controlled trial. Am J Kidney Dis. 2014;64:111-8.

7. Wizemann $V$, Wabel $P$, Chamney $P$, et al. The mortality risk of overhydration in haemodialysis patients. Nephrol Dial Transplant. 2009;24:1574-9.

8. Onofriescu M, Siriopol D, Voroneanu L, et al. Overhydration, cardiac function and survival in hemodialysis patients. PLoS One. 2015;10(8):e0135691.

9. Zoccali C, Moissl U, Chazot C, et al. Chonic fluid overload and mortality in ESRD. J Am Soc Nephrol. 2017:28:2491-7.

10. Daugirdas JT, Schneditz D. Overestimation of hemodialysis dose depends on dialysis efficiency by regional blood flow but not by conventional two pool urea kinetic analysis. ASAIO J. 1995;41:M719-24.

11. Leypoldt JK. Urea standard Kt/N (urea) for assessing dialysis treatment adequacy. Hemodial Int. 2004;8:193-7.

12. Movilli E, Gaggia P, Zubani R, et al. Association between high ultrafiltration rates and mortality in uraemic patients on regular haemodialysis. A 5-year prospective observational multicentre study. Nephrol Dial Transplant. 2007; 22:3547-52.

13. Assimon MM, Wenger JB, Wang L, Flythe JE. Ultrafiltration rate and mortality in maintenance hemodialysis patients. Am J Kidney Dis. 2016:68:911-22.

14. Katzarski KS, Charra B, Luik AJ, et al. Fluid state and blood pressure control in patients treated with long and short haemodialysis. Nephrol Dial Transplant. 1999;14:369-75.

15. Zoccali C, Torino C, Tripepi R. Et al; lung US in CKD working group. Pulmonary congestion predicts cardiac events and mortality in ESRD. J Am Soc Nephrol. 2013;24:639-46

16. Torino C, Gargani L, Sicari R, et al. The agreement between auscultation and lung ultrasound in hemodialysis patients: the LUST study. Clin J Am Soc Nephrol. 2016:11:2005-11.

17. Loutradis C, Sarafidis PA, Ekart R, et al. The effect of dry-weight reduction guided by lung ultrasound on ambulatory blood pressure in hemodialysis patients: a randomized controlled trial. Kidney Int. 2019;95:1505-13.

18. Gargani $L$, Sicari R, Raciti M, et al. Efficacy of a remote web-based lung ultrasound training for nephrologists and cardiologists: a LUST trial subproject. Nephrol Dial Transplant. 2016;31:1982-8.

19. Garg AX, Suri RS, Eggers $P$, et al. Patients receiving frequent hemodialysis have better health-related quality of life compared to patients receiving conventional hemodialysis. Kidney Int. 2017:91:746-75.

20. Eneanya ND, Maddux DW, Reviriego-Mendoza MM, et al. Longitudinal patterns of health-related quality of life and dialysis modality: a national cohort study. BMC Nephrol. 2019;20:7

\section{Publisher's Note}

Springer Nature remains neutral with regard to jurisdictional claims in published maps and institutional affiliations.

Ready to submit your research? Choose BMC and benefit from

- fast, convenient online submission

- thorough peer review by experienced researchers in your field

- rapid publication on acceptance

- support for research data, including large and complex data types

- gold Open Access which fosters wider collaboration and increased citations

- maximum visibility for your research: over $100 \mathrm{M}$ website views per year

At $B M C$, research is always in progress.

Learn more biomedcentral.com/submissions 